\title{
Relationship between pulse width and energy in GRB 060124: from X-ray to gamma-ray bands
}

\author{
Fu-Wen Zhang ${ }^{\mathrm{a}, \mathrm{b}}$, Yi-Ping Qin ${ }^{\mathrm{c}, \mathrm{d}}$ \\ ${ }^{a}$ National Astronomical Observatories/Yunnan Observatory, Chinese Academy of \\ Sciences, P.O. Box 110, Kunming, Yunnan 650011, China \\ ${ }^{\mathrm{b}}$ The Graduate School of the Chinese Academy of Sciences, P.O. Box 3908, \\ Beijing 100039, China \\ ${ }^{\mathrm{c}}$ Center for Astrophysics, Guangzhou University, Guangzhou 510006, China \\ ${ }^{\mathrm{d}}$ Physics Department, Guangxi University, Nanning, Guangxi 530004, China
}

\begin{abstract}
GRB 060124 is the first event that both prompt and afterglow emission were observed simultaneously by the three Swift instruments. Its main peak also triggered Konus-Wind and HETE-II. Therefore, investigation on both the temporal and spectral properties of the prompt emission can be extended to X-ray bands. We perform a detailed analysis on the two well identified pulses of this burst, and find that the pulses are narrower at higher energies, and both X-rays and gamma-rays follow the same $w-E$ relation for an individual pulse. However, there is no a universal powerlaw index of the $w-E$ relation among pulses. We find also that the rise-to-decay ratio $r / d$ seems not to evolve with $E$ and the $r / d$ values are well consistent with that observed in typical GRBs. The broadband spectral energy distribution also suggest that the X-rays are consistent with the spectral behavior of the gamma-rays. These results indicates that the X-ray emission tracks the gamma-ray emission and the emissions in the two energy bands are likely to be originated from the same physical mechanism.
\end{abstract}

Key words: gamma-rays: bursts; method: statistical; X-rays: bursts; X-rays: individual (GRB 060124)

PACS: 95.85.Pw; 95.85.Nv

Email addresses: fwzhang@ynao.ac.cn (Fu-Wen Zhang), ypqin@ynao.ac.cn (Yi-Ping Qin). 


\section{Introduction}

The Swift satellite (Gehrels et al., 2004) was successfully launched on 20th November 2004. It is a multi-wavelength observatory, covering the gammaray, X-ray and UV/optical bands. Thanks to its rapid repointing capability, the mission has revolutionized the Gamma-Ray Burst (GRB) observations in many aspects (for recent reviews, see Mészáros, 2006; Fox and Mészáros, 2006; Zhang, 2007). The prompt slewing capability of the X-Ray Telescope (XRT, Burrows et al., 2005) and UV-Optical Telescope (UVOT, Roming et al., 2005) allows the satellite to swiftly catch very early X-ray and UV/optical signals following the GRB prompt emission detected by the Burst Alert Telescope (BAT, Barthelmy et al., 2005).

In the pre-Swift era, the temporal and spectral behaviors of GRB prompt emission have been studied extensively. It is found that a pulse of the prompt gamma-rays at lower energy bands tend to be wider, which is roughly depicted as $w \propto E^{-0.4}(w-E$ relation; Fishman et al., 1992; Link et al., 1993; Fenimore et al., 1995; Norris et al., 1996, 2005; Piro et al., 1998; Costa, 1999; Nemiroff, 2000; Feroci et al., 2001; Crew et al., 2003; Peng et al., 2006; Zhang et al., 2007b). The photons at lower energy bands also lag behind that of the photons at higher energy bands (the so-called spectral lag; Cheng et al., 1995; Norris et al., 1996, 2005; Norris, Marani and Bonnell, 2000; Wu and Fenimore, 2000; Chen et al., 2005; Yi et al., 2006; Zhang et al., 2006b, 2006c; Peng et al., 2007).

Although many attempts have been made to explain the $w-E$ relation and the spectral lag behavior(e.g. Fenimore et al. 1995; Cohen et al., 1997; Chiang, 1998; Dermer, 1998; Kazanas et al. 1998; Piran, 1999; Wang et al., 2000; Nemiroff, 2000; Qin et al., 2004, 2005; Shen et al., 2005; Lu et al., 2006; Zhang et al., 2007b; Dado, Dar and De Rújula, 2007), its nature remains a matter of active debate in the community. On the other hand, it is unclear whether this correlation can be extended to X-ray bands. The broadband observations showed that the X-ray emission of some GRBs have unusual properties. in't zand et al. (1999) found that the prompt X-ray emission of GRB 980519, measured by BeppoSAX, undergoes a strong soft-to-hard-to-soft evolution. An exceptionally intense gamma-ray burst, GRB 030329, was detected and localized by the instruments on board the High Energy Transient Explorer satellite (HETE). It's lightcurve has a distinct, bright, soft X-ray component(Vanderspek et al. 2004). A thermal emission component is identified from the XRT data of a nearby XRF 060218 (Campana et al. 2006), but its non-thermal X-rays are from the same emission component as the gamma-rays (Liang et al., 2006). Vetere et al. (2006) analyzed the X-ray temporal and spectral characteristics of ten GRBs detected by the WFCs on board BeppoSAX and argued that there exist two components (slow and fast) in the X-ray emission. These facts suggest that the physics of these prompt X-rays are also very uncertain. 
With benefit from the Swift satellite, the prompt X-rays of some bursts were observed, e.g. GRB 050117 (Hill et al., 2006), GRB050713A (Morris et al., 2007), GRB050820A (Cenko et al., 2006), GRB 060124 (Romano et al., 2006), GRB 060218 (Campana et al., 2006; Liang et al., 2006); GRB 061121 (Page et al., 2007) and GRB 070129( Godet et al., 2007; Krimm et al., 2007). These panchromatic observations unveiled the unprecedented spectral and temporal information of GRB prompt emission. This also makes it possible to measure their temporal structures and to examine whether the well-known $w-E$ relation can be extended to X-ray bands for these bursts. Except for GRB 060124 and 060218, these bursts have very complicated temporal structures that consist of a series of overlapping pulses. Liang et al. (2006) analyzed the non-thermal emission of GRB 060218 from the gamma-ray to X-ray bands and obtained that $w \propto E^{-0.31 \pm 0.03}$, which roughly satisfies the $w-E$ relation and the relation between spectral lag and luminosity derived from typical GRBs (Fenimore et al. 1995; Norris et al., 1996, 2000, 2005), although it has the longest pulse duration and spectral lag observed to date among the observed GRBs. They suggested that the prompt X-rays and gamma-rays are from the same component. In this paper we present a detailed analysis on the prompt emission of GRB 060124 to examine whether the $w-E$ relation can be extended to X-rays. In Section 2, we present the data reduction. Results are given in Section 3. Our conclusions are presented in Section 4.

\section{Data Reduction}

GRB 060124 was detected by Swift-BAT at 15:54:52 UT on 24 January 2006 (trigger 178750, Holland et al., 2006; Fenimore er al., 2006), located at right ascension $05^{h} 08^{m} 10^{s}$ and declination $+69^{0} 42^{\prime} 33^{\prime \prime}$, with an uncertainty of $3^{\prime}$. The burst also triggered Konus-Wind (Golenetskii et al., 2006) 559.351 s and the FREGATE instrument aboard HETE-II (HETE trigger 4012, Lamb et al., 2006) $557.7 \mathrm{~s}$ after the BAT trigger. The prompt emission was observed simultaneously by XRT $\left(T_{0}+104 \mathrm{~s}\right.$, where $T_{0}$ denotes the BAT trigger time) and UVOT at $V=16.96 \pm 0.08\left(T_{0}+183 \mathrm{~s}\right)$ and $V=16.79 \pm 0.04\left(T_{0}+633 \mathrm{~s}\right)$. Swift-BAT first triggered on a precursor, then after about $500 \mathrm{~s}$ three major peaks following the precursor were observed. The burst is one of the longest GRBs (even excluding the precursor) recorded by either BATSE or Swift, and it is the first event that three Swift instruments have a clear detection of both the prompt and the afterglow emission (Romano et al., 2006).

The main peak data of GRB 060124 are not included in the event data since BAT was triggered by the precursor of the burst and the event data lasted only $t \sim 300 \mathrm{~s}$ after the trigger. The main peaks of the burst are visible in the mask-tagged 4 channel (15-25, 25-50, 50-100 and 100-350 keV bands) light curves, where the time bin is $1.6 \mathrm{~s}$, generated by the flight software (see Fig. 1). 
The XRT observations in sequence 000 included a net exposure time of $t \sim 867$ $\mathrm{s}$ in Windowed Timing (WT) mode and $15 \mathrm{~s}$ in Photon Counting (PC) mode. The WT mode data recorded the main burst and were adopted in this work. The data were reduced with the standard XRTDAS tools, using the latest calibration files available in CALDB and the standard data screening. For our analysis, events in the 0.2-10 keV band with grades 0-2 were used (see Burrows et al. 2005). The data of the WT mode during sequence 000 were affected by pile-up. To account for this effect, we extract a rectangular $40 \times 20$-pixel region with a $4 \times 20$-pixel region excluded from its center as the source region. The background region is a box $(40 \times 20$-pixel $)$ far away from the source. The XRT-WT events were extracted in three energy bands, 0.2-1, 1-4 and 4-10 $\mathrm{keV}$ (the corresponding backgrounds were subtracted). These are also shown in Fig. 1. The count rates of the burst were also recorded by Konus in three energy ranges: G1 (18-70 keV), G2 (70-300 keV), and G3 (300-1160 keV). The Konus trigger data are recorded from $T_{K W}-0.512 \mathrm{~s}$ to $T_{K W}+229.632 \mathrm{~s}$ with time resolution ranging from 2 to $256 \mathrm{~ms}$. The data before $T_{K W}-0.512 \mathrm{~s}$ are collected in the waiting mode with $2.944 \mathrm{~s}$ time resolution. The Konus time history of this burst in the G3 band (2.944 s time resolution) is reported in Fig. 1 as well.

\section{Results}

As shown in Fig. 1, the largest peak ( 560-580 s) exists significantly in all eight energy bands. The temporal properties of this peak in both the X-ray and gamma-ray bands are worth discussing. This peak consists of three main pulses. We focus on two apparent pulses among them.

Kocevski et al. (2003) developed an empirical expression, which can be used to fit the pulses of GRBs. This function is written as

$$
F(t)=F_{m}\left(\frac{t+t_{0}}{t_{m}+t_{0}}\right)^{r}\left[\frac{d}{d+r}+\frac{r}{d+r}\left(\frac{t+t_{0}}{t_{m}+t_{0}}\right)^{(r+1)}\right]^{-\frac{r+d}{r+1}}
$$

where $t_{m}$ is the time of the maximum flux $\left(F_{m}\right)$ of the pulse, $t_{0}$ is the offset time, $r$ and $d$ are the rising and decaying power-law indices, respectively.

From the BAT light curves we find that the peak considered here is apparently separable at about $T_{0}+540 \mathrm{~s}$, but it is not separable in the three XRT energy ranges due to overlapping. Norris et al. (1996) developed a method to deconvolve an overlapped GRB temporal profile into pulses. We use this method deconvolve the temporal profiles of the burst from $450 \mathrm{~s}$ to $540 \mathrm{~s}$ into seven pulses in the three XRT energy bands, where Eq. 1 is adopted as the pulse model. An interactive graphical IDL routine and a least-squares algorithm are 
applied to the pulse fit. The front pulses obtained from the fit are subtracted from the XRT light curves, which yields new data of the light curves. In this way, the peaks after $540 \mathrm{~s}$ are no more affected by the front overlapping pulses. Presented in Fig. 2 are these new XRT light curves and the BAT light curves.

It is known that the burst temporal profiles are self-similar across energy bands (e.g. Norris et al. 1996). We deconvolve the largest peaks from $540 \mathrm{~s}$ to $650 \mathrm{~s}$ into three pulses for all XRT and BAT energy bands with the same methods described above, where the new XRT data are adopted. The fitting results are shown in Fig. 2 as wel 1 . We label the two apparent pulses in the concerned energy bands as Pulse 1 (that with the smaller magnitude) and Pulse 2 (that with the larger magnitude), respectively (see Fig. 2).

The data recorded by Konus before $T_{K W}-0.512 \mathrm{~s}$ are collected in the waiting mode with $2.944 \mathrm{~s}$ time resolution. Since this time resolution is low, the corresponding data cannot be used in our analysis. We adopt only the $256 \mathrm{~ms}$ trigger data after $T_{K W}-0.512 \mathrm{~s}(558.839 \mathrm{~s}$ after the BAT trigger) to preform the temporal analysis in the $300-1160 \mathrm{keV}$ band. The data are also displayed in Fig. 2. We find from the figure that, in the $300-1160 \mathrm{keV}$ band, only one pulse (Pulse 2) can be identified and can be fitted with Eq. 1.

We measure the pulse width $w$, and the ratio of the rising time to the decaying time $r / d$ at the full-width half-maximum (FWHM) of the fitting curves. The uncertainties of these quantities are derived from the errors of the fitting parameters according to the error transform function. The results are listed in Table 1, where the errors are reported in the $1 \sigma$ confidence level.

There exists a significant trend that pulses are narrower at higher energies. The $w-E$ relations for two pulses are presented in Fig. 3, where $E$ is the geometric mean of the lower and upper boundaries of the energy band. It shows that the two quantities are correlated. Following Fenimore et al. (1995), we parameterize the dependence of the pulse width on energy by a power law. The best fit yields $w \propto E^{-0.47 \pm 0.05}(\mathrm{~N}=5)$ for Pulse 1 and $w \propto E^{-0.23 \pm 0.03}$ $(\mathrm{N}=6)$ for Pulse 2. Please note that the data in $0.2-1$ and $1-4 \mathrm{keV}$ bands are not included in the fits. It is found that the power-law index for Pulse 1 is roughly consistent with that previously observed in typical GRBs, but it is much shallower for Pulse 2, similar to that observed in GRB 060218 (Liang et al., 2006). Note that the distribution of the index for a typical GRB sample has a large dispersion, with a median of $\sim-0.4$ (see, Jia and Qin, 2005; Peng et al., 2006; Zhang et al., 2007b). Thus, it is possible that there is no a universal power-law index of the $w-E$ relation. For these two pulses, we find that their $r / d$ values are well consistent with that observed in typical GRBs (e.g.,

1 Due to the heavily overlapping in $0.2-1 \mathrm{keV}$ and $1-4 \mathrm{keV}$ bands, we cannot obtain very robust data within these bands from this analysis. Thus, parameters derived from these two bands are regarded as merely qualitative results. 
Norris et al., 1996, 2005; Liang et al., 2002; Peng et al., 2006), but no apparent relationship between $r / d$ and $E$ is observed.

Broadband spectral energy distribution (SED) is also helpful to discriminate different emission components. We derive the SED from the peak fluxes of an individual pulses at different energy bands by using the spectral data 2 . The results are listed in Table 2 (also see Fig. 4). From Table 2 and Fig. 4, one can find that the peak fluxes for the two pulses become larger at higher energies, suggesting that the X-rays are the same emission component of the gamma-rays.

\section{Conclusions and Discussion}

We have analyzed the temporal properties of the two well-identified pulses of GRB 060124 from X-ray $(0.2-10 \mathrm{keV})$ to gamma-ray $(15-1160 \mathrm{keV})$ energy bands. We find that the pulse width $w$ is energy-dependent for the two pulses in eight energy bands (0.2-1, 1-4, 4-10, 15-25, 25-50, 50-100, 100-350 and 300$1160 \mathrm{keV}$ ). The pulses are found narrower at higher energies, and both $\mathrm{X}$ rays and gamma-rays follow the same $w-E$ relation for an individual pulse. However, we find that there is no a universal power-law index of the $w-E$ relation among pulses. We also find that the rise-to-decay ratio $r / d$ seems not to evolve with $E$ and the $r / d$ values are well consistent with that observed in typical GRBs. The peak fluxes for the two pulses become larger at higher energies. These results indicates that the X-ray emission tracks the gamma-ray emission and the emissions in the two energy bands are likely to be originated from the same physical mechanism.

One remarkable advance from Swift is that the on-board XRT has established a large sample of X-ray lightcurves from tens of seconds to days (Zhang et al., 2006a; Nousek et al., 2006; O'Brien et al., 2006). The physical mechanisms of these X-rays are of great uncertain and is on debate in the GRB community (see review by Zhang, 2007). It is possible that the mechanisms are diverse (e.g., Zhang et al., 2006a; Zhang et al., 2007a, Liang et al., 2007, 2008). As we show here that some X-rays are possibly from the same emission component (see also Liang et al., 2006 for GRB 060218). However, Vetere et al. (2006) analyzed the temporal and spectral characteristics of X-rays for ten GRBs detected by the WFCs on board BeppoSAX and argued that there exist two components (slow and fast) in the X-ray emission. This feature actually is also seen in GRB 030329 (Vanderspek et al., 2004). Both temporal and spectral properties are critical to discriminate these components.

2 Due to the spectral data observed by Konus-Wind are not provided, the peak flux of Pulse 2 in $300-1160 \mathrm{keV}$ is not measured. 


\section{Acknowledgments}

We are grateful to Dr. Valentin Pal'shin for providing the Konus-Wind data.

We should also like to thank the anonymous referee for helpful suggestions and En-Wei Liang, Jin-Ming Bai and Bin-Bin Zhang for valuable discussion. This work is supported by National Natural Science Foundation of China (No. 10573030, No. 10533050 and No. 10463001).

\section{References}

Barthelmy, S. D., et al., 2005. Spa. Sci. Rev. 120, 143.

Burrows, D. N., et al., 2005. Spa. Sci. Rev. 120, 165.

Campana, S., et al., 2006. Nature 442, 1008.

Cenko, S. B., Kasliwal, M., Harrison, F. A., et al., 2006. ApJ 652, 490.

Chen, L., Lou, Y.-Q., Wu, M., Qu, J.-L., Jia, S.-M., Yang, X.-J., 2005. ApJ $619,983$.

Cheng, L. X., Ma, Y. Q., Cheng, K. S., Lu, T., Zhou, Y. Y., 1995. A\&A 300, 746.

Chiang, J., 1998. ApJ 508, 752.

Cohen, E., Katz, J. I., Piran, T., Sari, R., Preece, R. D., Band, D. L., 1997. ApJ 488, 330.

Costa, E., 1999. Nucl. Pphs. B (Proc. Suppl.) 69, 646.

Crew, G. B., et al., 2003. ApJ 599, 387.

Dado, S., Dar, A., De Rújula, A., 2007. ApJ, 663, 400.

Dermer, C. D., 1998. ApJ 501, L157.

Fenimore, E. E., in 't Zand, J. J. M., Norris, J. P., Bonnell, J. T., Nemiroff, R. J., 1995. ApJ 448, L101.

Fenimore, E., et al., 2006. GCN Circulars 4586.

Feroci, M., et al., 2001. A\&A 378, 441.

Fishman, G. J., Meegan, C. A., Wilson, R. B., Horack, J. M., Brock, M. N., Paciesas, William S., Pendleton, G. N., Kouveliotou, C., 1992. in AIP Conf. Proc. 265, Gamma-Ray Bursts, ed. W. S. Paciesas \& G. J. Fishman (New York: AIP), 13.

Fox, D. B., Mészáros, P., 2006. NJPh 8, 199.

Gehrels, N., et al., 2004. ApJ 611, 1005.

Godet, O., et al., 2007. GCN Circular 6053.

Golenetskii, S., Aptekar, R., Mazets, E., Pal'shin, V., Frederiks, D., Cline, T., 2006. GCN Circular 4599.

Hill, J. E., et al., 2006. ApJ 639, 303.

Holland, S. T., et al., 2006. GCN Circulars 4570.

in't Zand, J. J. M., Heise, J., van Paradijs, J., Fenimore, E. E., 1999. ApJ 516, L57.

Jia, L.-W., Qin, Y.-P., 2005. ApJ 631, L25. 
Kazanas, D., Titarchuk, L. G., Hua, X.-M., 1998. ApJ 493, 708.

Kocevski, D., Ryde, F., Liang, E., 2003. ApJ 596, 389.

Krimm, H., et al., 2007. GCN Circular 6058.

Lamb, D., Ricker, G., Atteia, J-L., Kawai, N., Woosley, S., 2006. GCN Circulars 4601.

Liang, E. W., Xie, G. Z., Su, C. Y., 2002. PASJ 54, 1.

Liang, E. W., Zhang, B. B., Stamatikos, M., Zhang, B., Norris, J., Gehrels, N., Zhang, J., Dai, Z. G., 2006. ApJ 653, L81.

Liang, E.-W., Zhang, B.-B., Zhang, B., 2007. ApJ 670, 565.

Liang, E.-W., Racusin, J. L., Zhang, B., Zhang, B.-B., Burrows, D. N., 2008. ApJ, in press (arXiv:0708.2942).

Link, B., Epstein, R. I., Priedhorsky, W. C., 1993. ApJ 408, L81.

Lu, R.-J., Qin, Y.-P., Zhang, Z.-B., Yi, T.-F., 2006. MNRAS 367, 275.

Mészáros, P., 2006. Rev. Prog. Phys. 69, 2259.

Morris, D. C., Reeves, J., Pal'shin, V., et al., 2007. ApJ 654, 413.

Nemiroff, R. J., 2000. ApJ 544, 805.

Norris, J. P., Nemiroff, R. J., Bonnell, J. T., Scargle, J. D., Kouveliotou, C., Paciesas, W. S., Meegan, C. A., Fishman, G. J., 1996. ApJ 459, 393.

Norris, J. P., Marani, G. F., Bonnell, J. T., 2000. ApJ 534, 248.

Norris, J. P., Bonnell, J. T., Kazanas, D., Scargle, J. D., Hakkila, J., Giblin, T. W., 2005. ApJ 627, 324.

Nousek, J. A., et al., 2006. ApJ 642, 389.

O'Brien, P. T., et al., 2006. ApJ 647, 1213.

Page, K. L., et al., 2007. ApJ 663, 1125.

Peng, Z.-Y., Lu, R.-J., Qin, Y.-P., Zhang, B.-B, 2007. ChJAA 7, 428.

Peng, Z.-Y., Qin, Y.-P., Zhang, B.-B., Lu, R.-J. Jia, L.-W., Zhang, Z.-B., 2006. MNRAS 368, 1351.

Piran, T., 1999. Phys. Rep. 314, 575.

Piro, L., et al., 1998. A\&A 329, 906.

Qin, Y.-P., Zhang, Z.-B., Zhang, F.-W., Cui, X.-H., 2004. ApJ 617, 439.

Qin, Y.-P., Dong, Y.-M., Lu, R.-J., Zhang, B.-B., Jia, L.-W., 2005. ApJ 632, 1008.

Romano, P., et al., 2006. A\&A 456, 917.

Roming, P. W. A., et al., 2005. Spa. Sci. Rev. 120, 95.

Shen, R.-F., Song, L.-M., Li, Z., 2005. MNRAS 362, 59.

Vanderspek, R., et al., 2004. ApJ 617, 1251.

Vetere, L., Massaro, E., Costa, E., Soffitta, P., Ventura, G., 2006. A\&A 447, 499.

Wang, J. C., Cen, X. F., Qian, T. L., Xu, J., Wang, C. Y., 2000. ApJ 532, 267.

Wu, B., Fenimore, E., 2000. ApJ 535, L29.

Yi, T. F., Liang, E. W., Qin, Y. P., Lu, R. J., 2006. MNRAS 367, 1751.

Zhang, B., Fan, Y. Z., Dyks, J., Kobayashi, S., Mészáros, P., Burrows, D. N., Nousek, J. A., Gehrels, N., 2006a. ApJ 642, 354.

Zhang, B., 2007. ChJAA 7, 1. 
Zhang, B.-B., Liang, E.-W., Zhang, B., 2007a. ApJ 666, 1002.

Zhang, F.-W., Qin, Y.-P., Zhang, B.-B., 2007b. PASJ 59, 857.

Zhang, Z.-B., Deng, J.-G., Lu, R.-J., Gao, H.-F., 2006b. ChJAA 6, 312.

Zhang, Z., Xie, G. Z., Deng, J. G., Jin, W., 2006c. MNRAS 373, 729. 


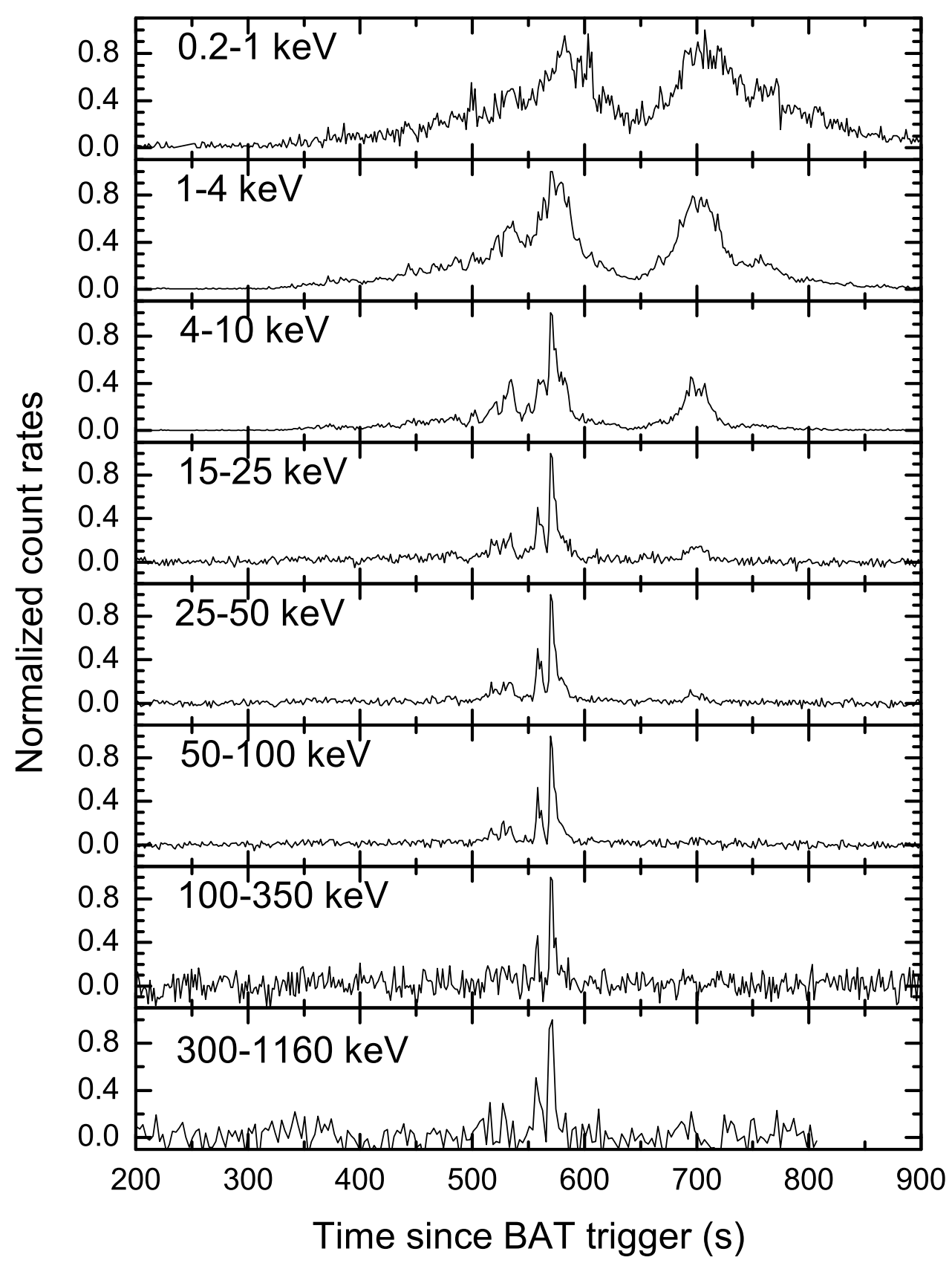

Fig. 1. XRT, BAT and Konus light curves of the prompt emission of GRB 060124.

The count rates have been normalized to the peak of each light curve. 


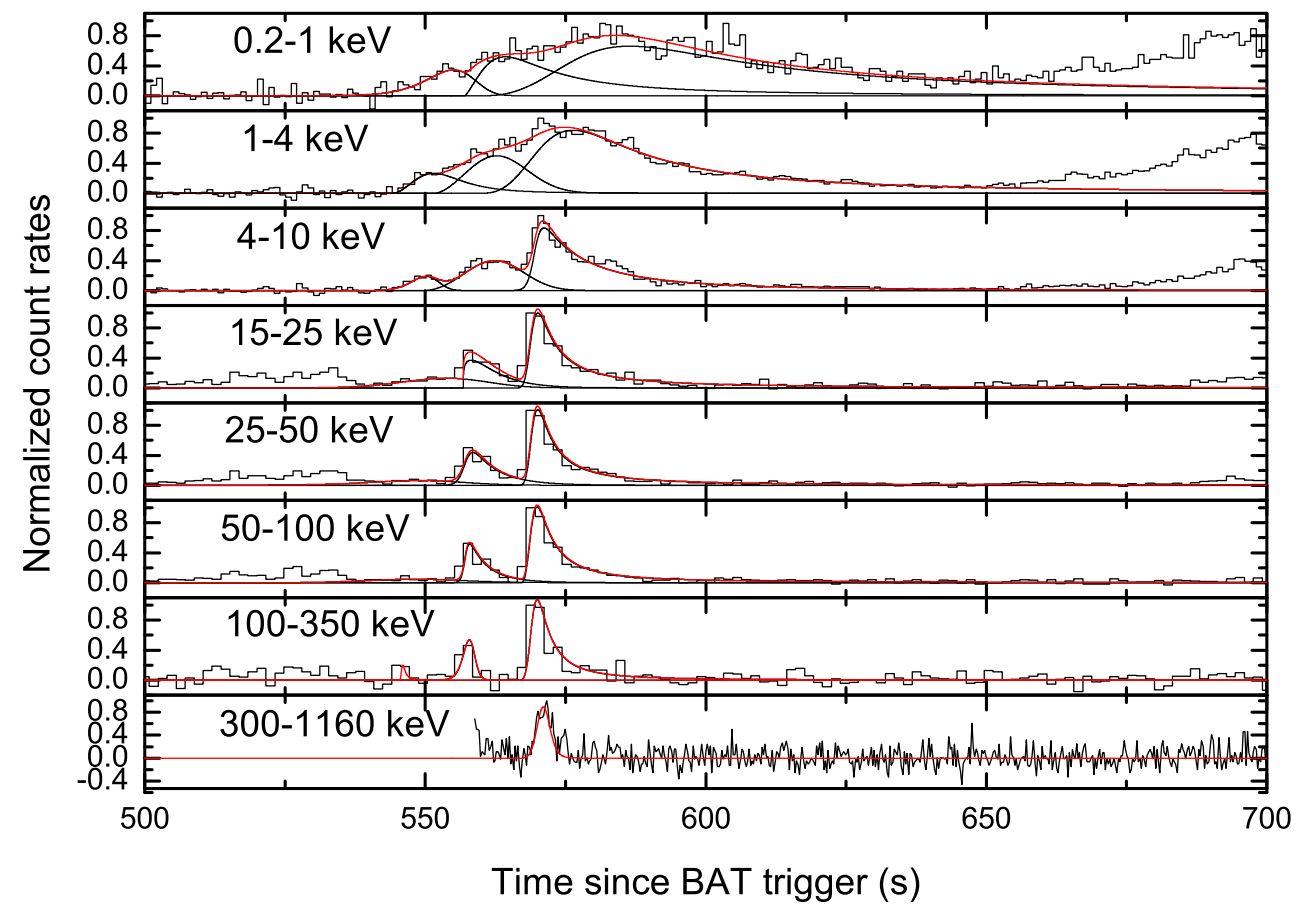

Fig. 2. XRT, BAT and Konus light curves around the largest flux of the burst, where the effects on the XRT light curves after $540 \mathrm{~s}$ by the front overlapping pulses have been checked. The three fitting pulses accounting for the largest peak (540-650 s) are also plotted in this figure. The red lines represent the superpositions of the three fitting pulses (in the $300-1160 \mathrm{keV}$ band, only one fitting pulse is adopted). 


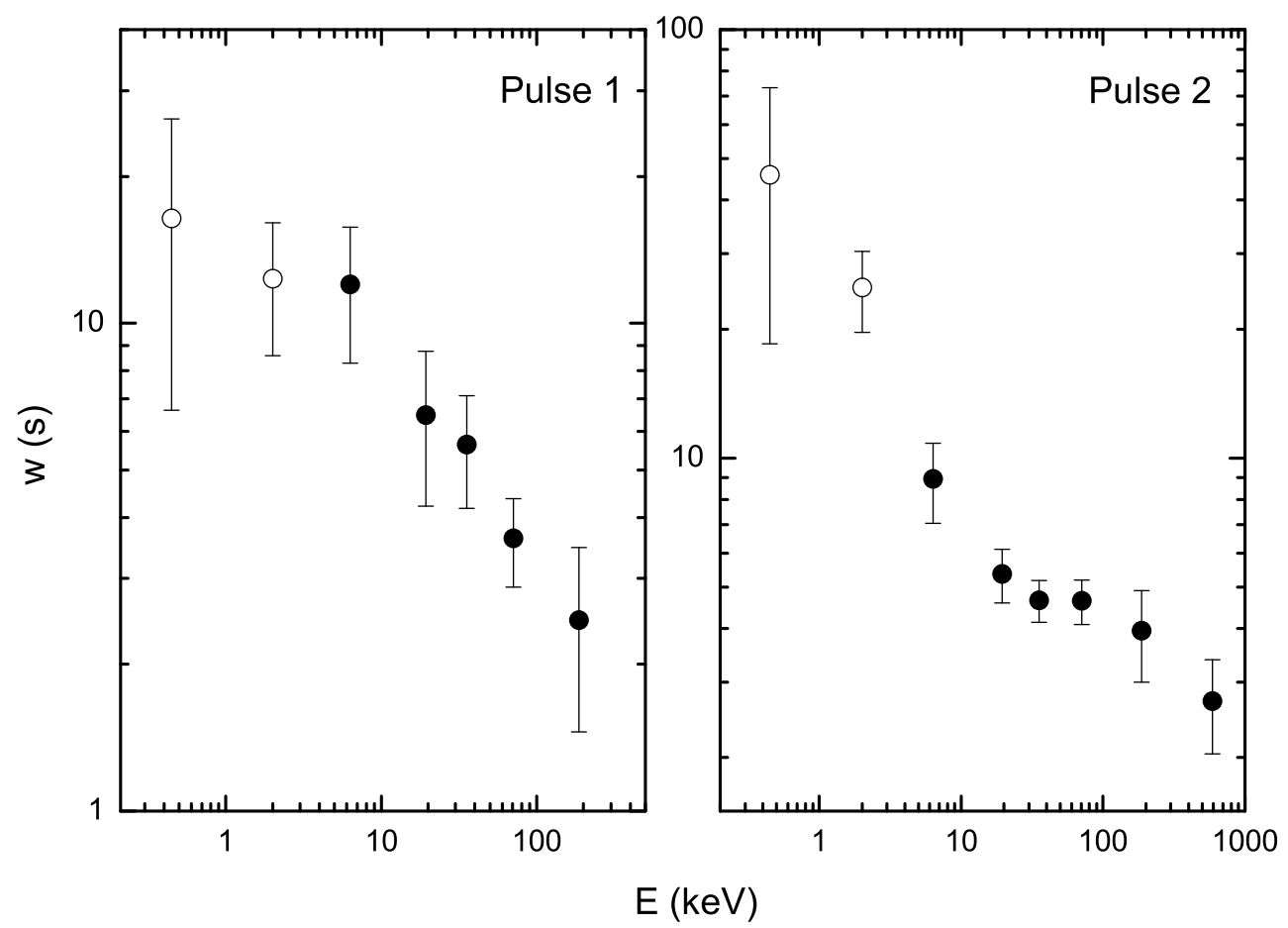

Fig. 3. Relationship between $w$ and $E$, where open circles denote $w$ estimated in 0.2-1 and 1-4 keV bands, and filled circles denote $w$ in other energy bands. 

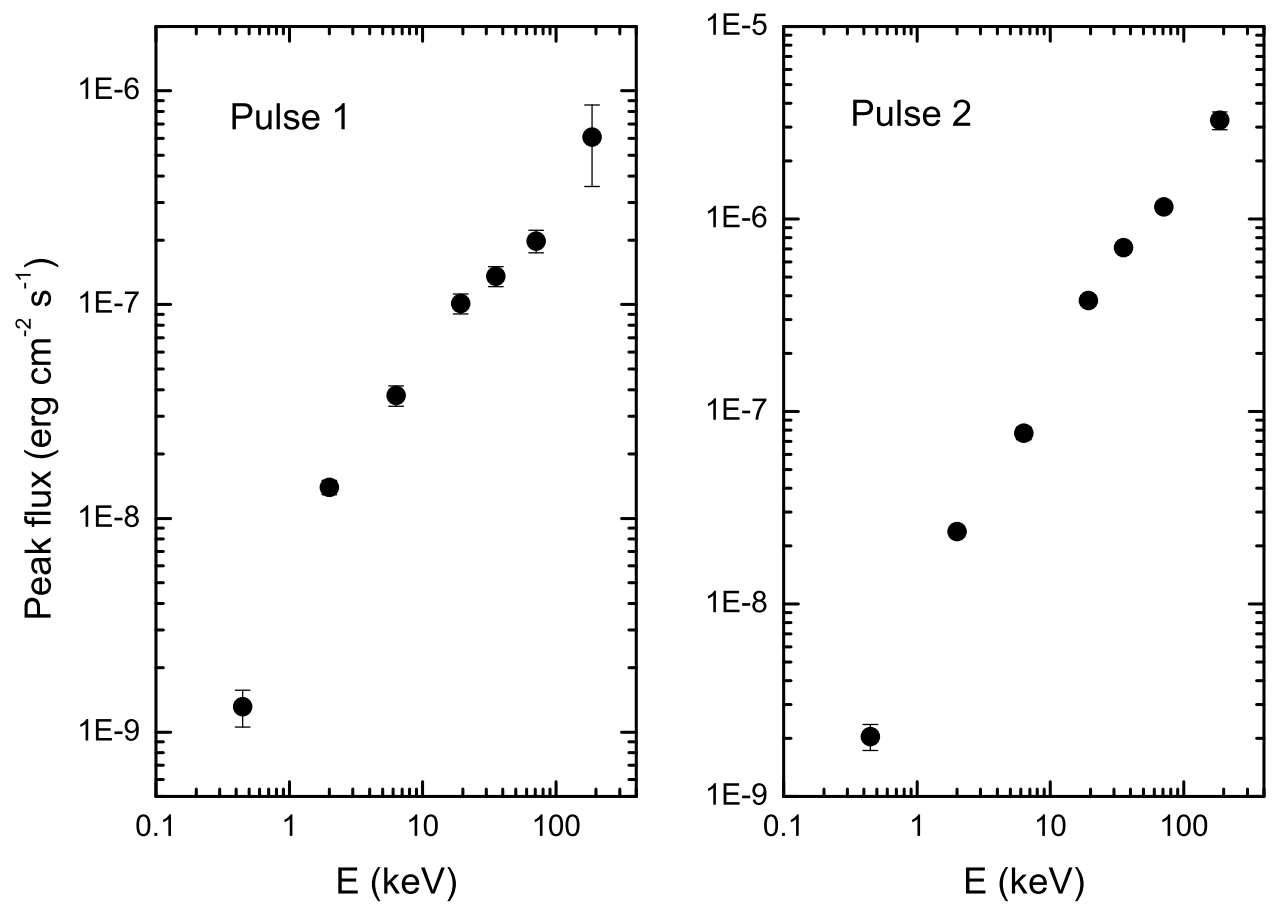

Fig. 4. Relationship between the pulse peak flux and energy. 
Table 1

Multi-wavelength temporal characteristics of the two pulses in GRB 060124.

\begin{tabular}{lcccc}
\hline \hline Band (keV) & Pulse 1 & \multicolumn{3}{c}{ Pulse 2} \\
& $\mathrm{w}(\mathrm{s})$ & $\mathrm{r} / \mathrm{d}$ & $\mathrm{w}(\mathrm{s})$ & $\mathrm{r} / \mathrm{d}$ \\
\hline $0.2-1$ & $16.4 \pm 9.8$ & $0.41 \pm 0.17$ & $45.8 \pm 27.4$ & $0.40 \pm 0.10$ \\
$1-4$ & $12.3 \pm 3.7$ & $0.50 \pm 0.31$ & $25.0 \pm 5.3$ & $0.45 \pm 0.09$ \\
$4-10$ & $12.0 \pm 3.7$ & $0.48 \pm 0.34$ & $8.9 \pm 1.9$ & $0.27 \pm 0.09$ \\
$15-25$ & $6.5 \pm 2.3$ & $0.26 \pm 0.15$ & $5.4 \pm 0.8$ & $0.38 \pm 0.14$ \\
$25-50$ & $5.6 \pm 1.5$ & $0.44 \pm 0.20$ & $4.7 \pm 0.5$ & $0.40 \pm 0.14$ \\
$50-100$ & $3.6 \pm 0.7$ & $0.36 \pm 0.20$ & $4.6 \pm 0.5$ & $0.42 \pm 0.15$ \\
$100-350$ & $2.5 \pm 1.0$ & $0.49 \pm 0.32$ & $4.0 \pm 1.0$ & $0.47 \pm 0.26$ \\
$300-1160$ & $\ldots$ & $\ldots$ & $2.7 \pm 0.7$ & $0.48 \pm 0.24$ \\
\hline
\end{tabular}

Table 2

Peak fluxes of the individual pulses at different energy bands in GRB 060124 .

\begin{tabular}{ccc}
\hline \hline & Pulse 1 & Pulse 2 \\
$\begin{array}{c}\text { Energy band } \\
(\mathrm{keV})\end{array}$ & $\begin{array}{c}\text { Peak energy flux } \\
\left(\mathrm{erg} \mathrm{cm}^{-2} \mathrm{~s}^{-1}\right)\end{array}$ & $\begin{array}{c}\text { Peak energy flux } \\
\left(\mathrm{erg} \mathrm{cm}^{-2} \mathrm{~s}^{-1}\right)\end{array}$ \\
\hline $0.2-1$ & $(1.3 \pm 0.3) \times 10^{-9}$ & $(2.1 \pm 0.3) \times 10^{-9}$ \\
$1-4$ & $(1.4 \pm 0.1) \times 10^{-8}$ & $(2.4 \pm 0.1) \times 10^{-8}$ \\
$4-10$ & $(4.8 \pm 0.4) \times 10^{-8}$ & $(7.7 \pm 0.6) \times 10^{-8}$ \\
$15-25$ & $(1.0 \pm 0.1) \times 10^{-7}$ & $(3.8 \pm 0.2) \times 10^{-7}$ \\
$25-50$ & $(1.4 \pm 0.1) \times 10^{-7}$ & $(7.1 \pm 0.2) \times 10^{-7}$ \\
$50-100$ & $(2.0 \pm 0.2) \times 10^{-7}$ & $(1.2 \pm 0.1) \times 10^{-6}$ \\
$100-350$ & $(6.1 \pm 0.3) \times 10^{-7}$ & $(3.3 \pm 0.3) \times 10^{-6}$ \\
\hline
\end{tabular}

